\title{
Strategies for Clinical Decision Support for Electronic Case Reporting
}

\author{
Noam H. Arzt ${ }^{1}$, Maiko Minami ${ }^{1}$, Daryl Chertcoff ${ }^{1}$, Janet Hui ${ }^{2}$ \\ ${ }^{1}$ HLN Consulting, LLC, Palm Desert, California, United States, ${ }^{2}$ Council of State and Territorial Epidemiologists, Atlanta, Georgia, United States \\ Objective
}

To discuss how clinical decision support (CDS) for electronic case reporting (eCR) will evolve over time to provide multiple deployment models

\section{Introduction}

As the knowledge required to support case reporting evolves from unstructured to more structured and standardized formats, it becomes suitable for electronic clinical decision support (CDS). CDS for case reporting confronts two challenges: a) While EHRs are moving toward local CDS capabilities, it will take several years for EHR systems to consistently support this capability; and b) public health-related CDS knowledge, such as Zika infection detection and reporting rules, may differ from jurisdiction to jurisdiction. Therefore, there is an ongoing need to manage reporting rules in a distributed manner. Similarly, there is a need for more decentralized models of CDS execution to overcome some of the disadvantages of centralized deployment and to leverage local CDS capabilities as they become available over the next several years.

\section{Methods}

The Reportable Condition Knowledge Management System (RCKMS) is a project funded by the CDC, through the Council of State and Territorial Epidemiologists (CSTE), to develop a tool that allows jurisdictions to author rules that define whether a patient is reportable for certain conditions. RCKMS includes a Decision Support Service (DSS) that runs the jurisdictions' rules and determines if a patient is reportable, for which condition(s) and to which jurisdiction(s). RCKMS currently plays a significant role in the broader Digital Bridge project that has been working to provide structure and governance around the national planning and implementation effort of eCR. RCKMS is currently a centralized CDS service that can be accessed by EHRs until they all have local CDS capabilities; and a knowledge authoring environment that allows ongoing distributed rule authoring. RCKMS supports the strategy for public health knowledge management, and it will evolve over time to provide the systems and services to satisfy short-, mid-, and long-term public health CDS requirements. In addition, RCKMS will comply with emerging technical standards that support this work.

\section{Results}

RCKMS is currently being deployed as a single, central, national service on the APHL Informatics Messaging Services (AIMS) platform, which is operated and maintained by Association of Public Health Laboratories (APHL). The AIMS platform connects directly with reporters and provides a routing and validation service for incoming and outgoing messages. Two distributed CDS scenarios for decentralized eCR models have been identified. In the first scenario, the Decision Support Service component of the RCKMS software is installed within a clinical organization (as it would be in a centralized service) and executed locally. The second distributed CDS scenario for eCR involves the distribution of the Reporting Specification rules without the software. In this scenario, local electronic health record (EHR) implementations would be required to consume the Reporting Specifications and utilize them in a CDS capability within their EHR.

\section{Conclusions}

It is expected that given the diversity of organizations, systems, and architectures in the United States that multiple deployment scenarios for CDS for eCR will be simultaneously deployed for the foreseeable future. It cannot be stressed enough, however, that in all scenarios - centralized and distributed - there must be a centralized and uniform authoring of the Reporting Specification rules, since the specifications themselves originate from public health through a centralized process and must be administered nationally through a well-established process. It is also essential that all sites have all the rules available to them, since there may

SDS Annual Conference Proceedings 2019. This is an Open Access article distributed under the terms of the Creative Commons AttributionNoncommercial 4.0 Unported License (http://creativecommons.org/licenses/by-nc/3.0/), permitting all non-commercial use, distribution, and reproduction in any medium, provided the original work is properly cited. 
be multiple jurisdictions whose rules and reporting is required, with the determination of jurisdiction(s) based on where a patient lives and where they receive care.

\section{Acknowledgement}

This project is being conducted with support from the Centers for Disease Control and Prevention (CDC) through the Council of State and Territorial Epidemiologists (CSTE) 